\title{
Les images de satellite et les mesures de la couche de neige
}

Satellite pictures and snow measurements

\author{
Dominique Marbouty*, Charles Obled ** et Pierre Guillot***
}

\section{Les satellites et leurs instruments}

Nous nous intéresserons ici aux satellites actuellement utilisables pour une surveillance régulière de l'enneigement. Les caractéristiques importantes pour ce genre d'exploitation sont la résolution, la périodicité des images et les différents canaux disponibles: elles sont résumées dans le tableau I.

\begin{tabular}{|c|c|c|c|c|}
\hline \multicolumn{5}{|l|}{ Tableau I } \\
\hline Satellites & Radiomètre & $\begin{array}{c}\text { Canaux } \\
(\mathrm{en} \mu \mathrm{m})\end{array}$ & $\begin{array}{c}\text { Réso- } \\
\text { lution }\end{array}$ & $\begin{array}{c}\text { Périodicité } \\
\text { des images }\end{array}$ \\
\hline $\begin{array}{c}\text { NOAA } \\
\text { (Météorologie) }\end{array}$ & $\begin{array}{c}\text { VHRR } \\
\text { (Very High } \\
\text { Resolution }\end{array}$ & $\begin{array}{r}0.6-0.7 \\
10.5-12.5\end{array}$ & $1 \mathrm{~km}$ & 24 heures \\
& Radiometer) & & & \\
& MSS & $0.5-0.6$ & $80 \mathrm{~m}$ & \\
\hline LANDSAT & (Multi- & $0.6-0.7$ & $80 \mathrm{~m}$ & \\
(Etude des & Spectral & $0.7-0.8$ & $80 \mathrm{~m}$ & 18 jours \\
Ressources & Scanner) & $0.8-1.1$ & $80 \mathrm{~m}$ & \\
Terrestres) & 10.5-12.5 & $60 \mathrm{~m}$ & \\
\hline METEOSAT & & $0.4-1.1$ & $2,5 \mathrm{~km}$ & \\
(Géostationnaire) & & $5.7-7.1$ & $5 \mathrm{~km}$ & 25 minutes \\
& & $10.5-12.5$ & $5 \mathrm{~km}$ & \\
\hline
\end{tabular}

(*) Centre d'Etudes de la Neige (Météorologie Nationale) Grenoble.

(**) Institut de Mécanique de Grenoble

(***) Service "Ressources en Eau", Electricité de France (DTG) Grenoble.
Problèmes particuliers à la détection de la neige

\section{Distinction neige-nuage}

Avec les canaux les plus courants (visible et infrarouge thermique) cette distinction est souvent impossible. En effet la neige et les nuages ont à peu près la même réflectance dans le visible et, dans le cas de nuages sans grande extension verticale, étant à la même altitude, ils sont à la même température et ont donc des émissions dans l'infrarouge très voisines.

Les méthodes pour les distinguer sont donc très difficiles. Elles sont principalement de trois types :

- les nuages n'étant pas un phénomène fixe en général, on peut les éliminer en comparant plusieurs images successives. Ceci n'est possible que si l'on a une bonne périodicité. D'autre part les nuages orographiques peuvent poser des problèmes s'ils sont bien stables (Mac Clain et Baker, 1969),

- des méthodes d'étude des contours, de reconnaissance de forme permettent aussi d'éliminer ces nuages. Mais ceci est très coûteux et nécessite une très grande résolution,

- des études menées sur des données du Skylab EREP MSS ont montré que l'on pouvait discerner la neige des nuages à l'aide des trois canaux suivants : $0,4-0,5$; $0,7-0,8$ et surtout $1,55-175 \mu \mathrm{m}$ (Haefner, Itten, 1976). Ce canal n'existe sur aucun des satellites considérés, mais la prochaine série de Landsat (Follow on) en disposera avec le "Thematic mapper".

On voit donc que cette distinction pose de sérieux problèmes. De toute façon on n'a pas de données par temps couvert et on ne peut donc étudier le manteau neigeux que sur les zones dégagées. 


\section{La neige en forêt}

A l'exception de la période qui suit une précipitation neigeuse, c'est-à-dire tant que la neige reste accrochée sur les arbres, on ne peut pas distinguer, sur les images satellitaires, la neige dans les zones de forêt assez dense. Une solution consiste à regarder les clairières mais cela suppose une grande résolution, cela introduit des erreurs en période de fonte et enfin une telle méthode est très difficile à développer automatiquement.

Dans les grands bassins uniformément boisés à faible relief du Canada, Langham et Power (1977) proposent néanmoins de définir la limite régionale de la couverture neigeuse par un traitement statistique de la brillance des images de NOAA.

\section{Résolution nécessaire}

Elle dépend évidemment de ce que l'on veut étudier. Si l'on se réfère aux recommandations de l'OMM (International Working Seminar on Snow Studies by Satellites Geneva, Oct. 1976), les données VHRR ne peuvent pas être utilisées pour un bassin inférieur à 2 à $300 \mathrm{~km}^{2}$ et celles de Landsat permettent d'atteindre $10 \mathrm{~km}^{2}$.

Si l'on se place du point de vue de la détermination de l'altitude de la limite de la neige, le VHRR sous forme photographique permet une précision de $\pm 250 \mathrm{~m}$. Les données numériques permettent d'espérer, avec un traitement assez lourd, une précision de l'ordre de $\pm 100 \mathrm{~m}$ (si l'on néglige les problèmes exposés plus haut).

En général, pratiquement tous les chercheurs qui ont étudié ce problème sur le massif des Alpes l'ont fait à partir des données de Landsat (France, Suisse, Autriche). Les seuls travaux menés sur le VHRR ont été effectués en Suède et en France par les services météorologiques. Dans les deux cas, ils se heurtent à de gros problèmes de rectification d'images et surtout de résolution qui semble insuffisante sur des massifs très accidentés. Tout au moins les méthodes sont coûteuses en calcul pour des résultats encore assez décevants, tant qu'on ne dispose pas de relief numérisé des Alpes pour corriger les distorsions d'images.

\section{Périodicité des images}

Là aussi cela dépend de l'utilisation. Pour un travail de recherche ou de mise au point une seule image peut suffire. Par contre, s'il s'agit de suivre l'évolution du manteau neigeux sur un hiver, une image par semaine est nécessaire. Or, en fonction des problèmes posés par la nébulosité, pour atteindre ce résultat il semble nécessaire d'avoir une fréquence d'au moins une image par jour. On constate donc que pour un travail d'exploitation, les seuls satellites utilisables seraient NOAA ou Meteosat. C'est d'ailleurs ce que recommande l'OMM, en conseillant de mettre au point des modèles utilisant le VHRR et de se servir de Landsat pour caler ces modèles.

En conclusion,

- Landsat donne de très bons résultats grâce à sa résolution d'une part et au plus grand nombre de canaux d'autre part. Par contre, la périodicité de ces images ne permet pas un travail opérationnel ;
- NOAA permet un travail opérationnel mais assez grossier;

- Meteosat a bien une meilleure périodicité que NOAA mais une résolution un peu plus faible. Il est difficilement utilisable pour les zones à fort relief (Alpes, Pyrénées) à cause de l'incidence de la prise de vue $\grave{a}$ nos latitudes. Par contre il peut être intéressant pour les plateaux où cet effet est moins gênant.

Le problème semble donc difficile à résoudre car mème si l'on disposait d'un satellite à haute résolution avec image quotidienne (18 Landsat décalés par exemple) cela ferait une quantité énorme de données à traiter. Ce serait donc un gros effort à faire pour obtenir seulement la limite de la neige. Or cette limite en elle-même n'est pas une donnée capitale. Elle ne devient importante que si l'on arrive à la relier à une quantité d'eau disponible ce qui ne semble pas évident actuellement.

\section{Les propriétés radiatives et l'évolution de la couche de neige}

L'énergie solaire incidente, au niveau du sol, a un spectre assez bien connu, représenté figure 1 .

L'ensemble de l'énergie réfléchie, dans la bande $0.3-3.0 \mu \mathrm{m}$ permet de calculer l'albedo qui est la réflectance moyenne dans cette gamme de longueurs d'onde. On sait que l'albedo de la neige fraîche est voisin de 0.9 , mais en fait la réflectance varie en fonction de la longueur d'onde et, pour une longueur d'onde donnée, en fonction de l'état de surface (Fig. 2 et 3 ).

Les propriétés de réflectance de la neige sont développées par H.W. O'Brien et R.H. Munis (1975 et 1977). Elles peuvent d'ailleurs varier assez sensiblement avec l'angle sous lequel on effectue la mesure.

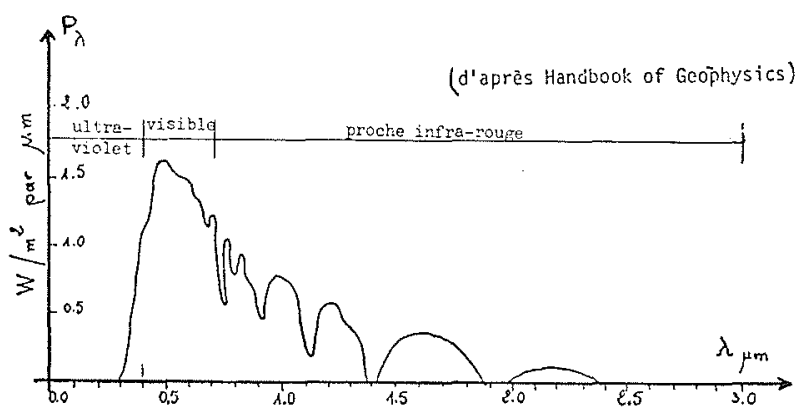

Figure 1 - Spectre du rayonnement solaire incident au niveau du sol.

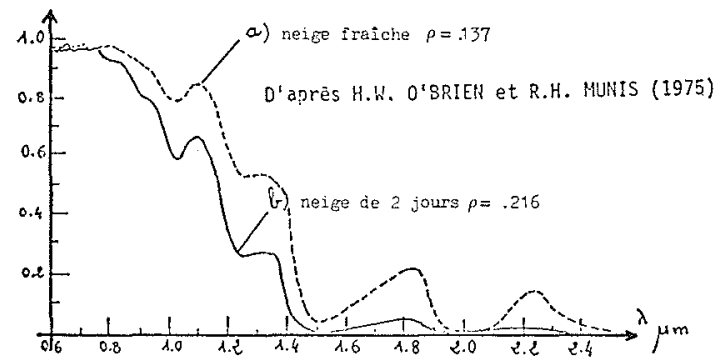

Figure 2 - Réflectance de la neige par rapport à $\mathrm{BaSO}_{4}$ 


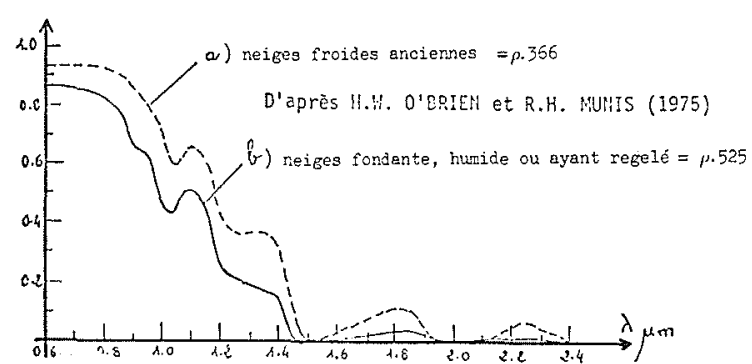

Figure 3 - Réflectance de la neige par rapport à $\mathrm{BaSO}_{4}$

Citons pour mémoire, le rayonnement, très faible, dans le domaine des micro-ondes, de $1 \mathrm{~mm}$ à $10 \mathrm{~cm}$ (fréquence de 100 à $1 \mathrm{GHz}$ ). Son intérêt essentiel est une grande variabilité en fonction de la teneur en eau liquide, mais sa mesure par satellite n'est envisageable qu'avec une résolution très grossière.

$\mathrm{Si}$ la neige a une réflectance assez élevée dans le visible, celle-ci décroît assez fortement dans l'infrarouge proche, ce pour de la neige fraîche. Le vieillissement par température négative a une influence faible dans le rouge et modéréee dans l'infrarouge, même s'il y a compaction par le vent. Par contre, la neige humide ou de la neige fondante ayant regelé, présente une réduction sensible de la réflectance dans le rouge et l'infrarouge. Cette différence permet de mettre en évidence les zones actives dans les processus de fusionregel.

Des résultats comparables peuvent se déduire des mesures de températures (infrarouge thermique): le sol découvert, pendant la journée a généralement une température positive, alors que toute la frange d'enneigement concernée par la fusion reste à $0^{\circ}$. Il est malheureusement impossible d'en déduire les intensités de fusion $(\mathrm{mm} / \mathrm{j})$. Ces images sont actuellement disponibles sur les satellites NOAA-4-VHRR qui explorent la bande $10.5-12.5 \mu \mathrm{m}$ avec une résolution voisine de $1 \mathrm{~km}$ (R.D. Seifert et al., 1975).

Une autre donnée intéressante pour 1'hydrologie est l'albedo de la neige. Comme on mesure l'énergie réfléchie, il suffit de calculer l'énergie potentielle incidente. A l'échelle à laquelle on travaille, celle-ci dépend, surtout par ciel clair, de la date de l'année et son spectre est assez bien connu pour une date donnée (cf. Cahier AFEDES). Par contre les mesures de l'énergie réfléchie sont assez imprécises car il y a saturation dans les bandes MSS 5 et 6. Mr. Mac Millan (1975) propose donc de n'utiliser que la bande MSS7 $(0.8 \cdot 1.1 \mu \mathrm{m})$, mais la réflectance calculée dans cette seule bande donne une valeur biaisée de l'albedo (en fait les études menées par la NASA (Rango, 1976) ont montré que le meilleur canal pour déterminer la limite de la neige est le canal $0.6-0.7 \mu \mathrm{m})$.

Mac Millan propose ensuite de lier l'albedo ou même directement la radiance à la densité moyenne du manteau. Les résultats, médiocres, s'expliquent par le fait que l'albedo est lié à un état de surface et que celui-ci n'est bien corrélé qu'à la densité de la couche superficielle (10 $\mathrm{cm}$ environ). Ce n'est donc pas un bon accès à l'équivalent en eau du manteau en tout point.

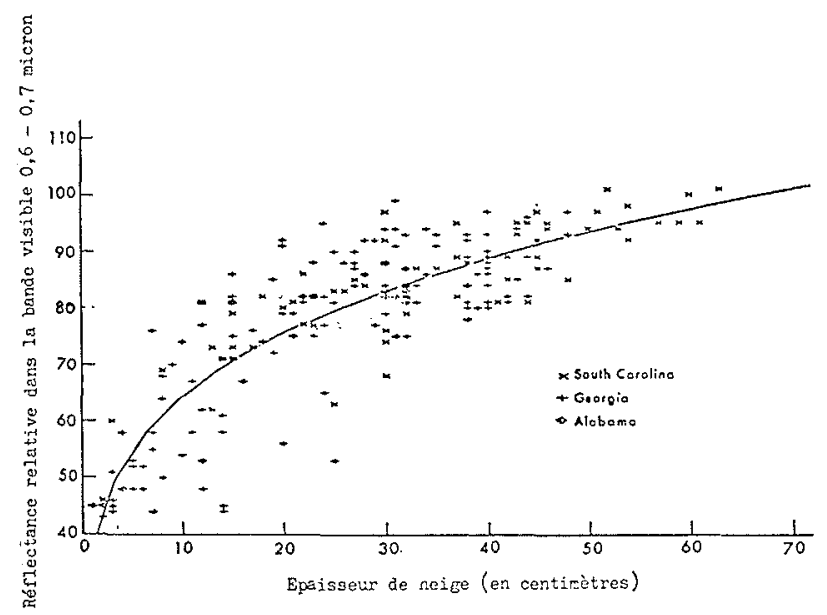

Figure 4 - D'après D.F. Mc Ginnis (1975)

Une autre approche, due à D.F. Mac Ginnis (1975), consiste à lier l'épaisseur de la couche à la brillance de la zone enneigée, ou à sa réflectance dans la bande visible de NOAA-2 $(0.6-0.7 \mu \mathrm{m})$. Pour une chute de neige donnée, et pour un nombre important de points, les hauteurs de neige au sol étaient disponibles d'où une possible analyse en corrélation :

$$
\begin{aligned}
& r^{2}=.74 \\
& N=201
\end{aligned}
$$

Il suffirait alors de quelques mesures de densité, car celle-ci a une faible variabilité spatiale, pour passer à la valeur en eau.

Mais la méthode appliquée à d'autres chutes de neige a donné des résultats plus discutables ( $r=0.4$ à 0.6 ), et il faut remarquer qu'elle s'applique à la première chute de neige, relativement faible $(10$ à $30 \mathrm{~cm})$, tombant sur un sol jusque-là découvert. En effet, au-delà de $30 \mathrm{~cm}$, il n'y a plus de variation sensible de la brillance, de même quand la chute recouvre un manteau existant. Cette méthode a donc un intérêt pour les grandes régions à enneigement épisodique, type plaine et plateau, et dépourvues de végétation haute (car sinon, les variations de brillance sont surtout liées à la végétation).

\section{Utilisation hydrologique de la surface enneigée}

On considère donc pour un bassin versant donné, la superficie enneigée ou le \% de surface enneigée. Toutefois l'imprécision sur la position de la ligne d'enneigement peut représenter une incertitude importante pour les petits bassins et il a fallu attendre une bonne résolution des images pour lever ce problème. Pratiquement, c'est avec Landsat-1 (lancé en juillet 1972), dont la résolution atteint $80 \mathrm{~m}$, que l'on a commencé à disposer de données assez fiables sur les superficies enneigées des petits bassins.

Compte tenu de la faible fréquence de passage des Landsat (18 jours) et des risques de ne pouvoir les uti- 
liser (couverture nuageuse, etc.), les premiers essais se sont limités à la prévision des apports saisonniers des grands bassins versants avec NOAA.

\section{Prévision de l'apport saisonnier}

\section{L'Indus à Attock (Pakistan)}

La figure 5 résume 1'article de A. Rango et V. Salomonson (1977).

La corrélation de l'apport des 4 mois avril-juillet avec la surface enneigée à la mi-avril (moyenne tirée chaque année entre le $1^{\text {er }}$ et le 20 avril des 3 meilleures photos de Nimbus de 1967 à 1972, de NOAA depuis 1973 avec une résolution de $4 \mathrm{~km}$ ) est de .903 , avec 5 observations, pour l'Indus à Besham $\left(162000 \mathrm{~km}^{2}\right)$ et de .945 , avec 7 observations, pour la Kaboul à Nowshera $\left(89000 \mathrm{~km}^{2}\right)$.

Les auteurs soulignent l'intérêt des données de satellite vu les difficultés d'obtention de mesures au sol dans ces bassins internationaux : mais - ce qu'ils oublient de signaler - la fonte commence début mars à cette latitude (Gulati, 1972) et leur prévision d'apport d'après les photos de satellite n'est disponible qu'après la miavril.

Pour la gestion des réservoirs de Tarbela et Kalabagh, on perd ainsi 2 mois : des prévisions d'après des mesures au sol seraient connues fin février. Car l'essentiel du stock neigeux est constitué dans ce bassin par les précipitations de janvier-février.

Wind River Mountains (Rango et Salomonson, 1975).

Il s'agit de 7 petits bassins des Rocheuses situés dans le Wyoming, de part et d'autre de la ligne de partage des eaux continentales.

Les seules données étant les photos Landsat-1 de 1973 et 1974, et les variables à expliquer les apports $Q$ du $15 \mathrm{mai}$ au 31 juillet, le faible effectif a conduit à mélanger les bassins pour faire nombre, en distinguant néanmoins deux classes, pour éviter la co-variation avec l'altitude. De même, pour éliminer la superficie du bassin, $Q$ est exprimé en débit spécifique par mille carré.

Les régressions sont représentées dans la figure 6 .

Cet exemple est peu convaincant : malgré les précautions prises, la covariation avec l'alimentation des bassins reste évidente. D'autre part la prévision est surtout intéressante si elle est disponible avant le $1^{\text {er }}$ avril, alors que les bassins sont encore enneigés à $100 \%$. Or ces méthodes supposent que $S$ soit déjà descendu à une valeur significativement différente de $100 \%$.
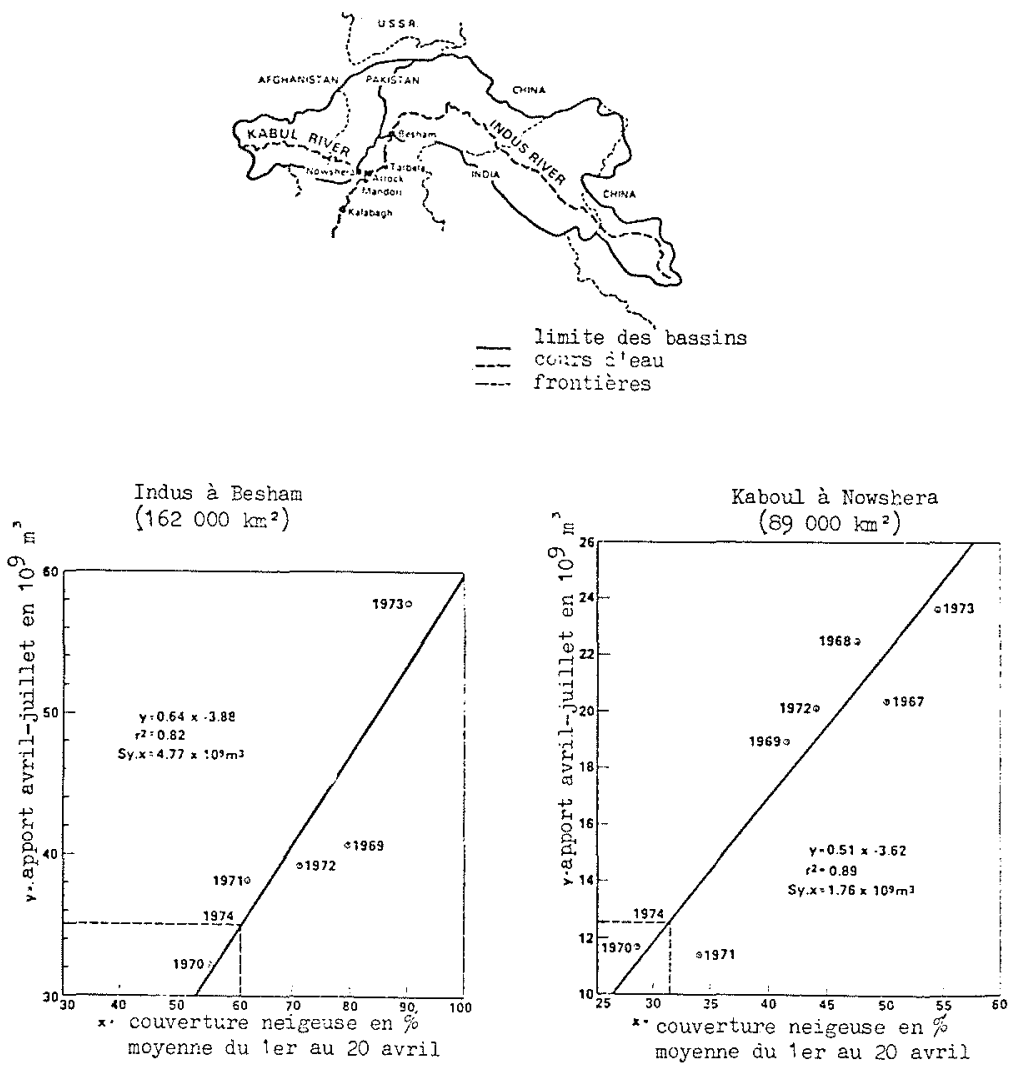

Figure 5 - (D'après Rango A. et V.V. Salomonson, 1977. 

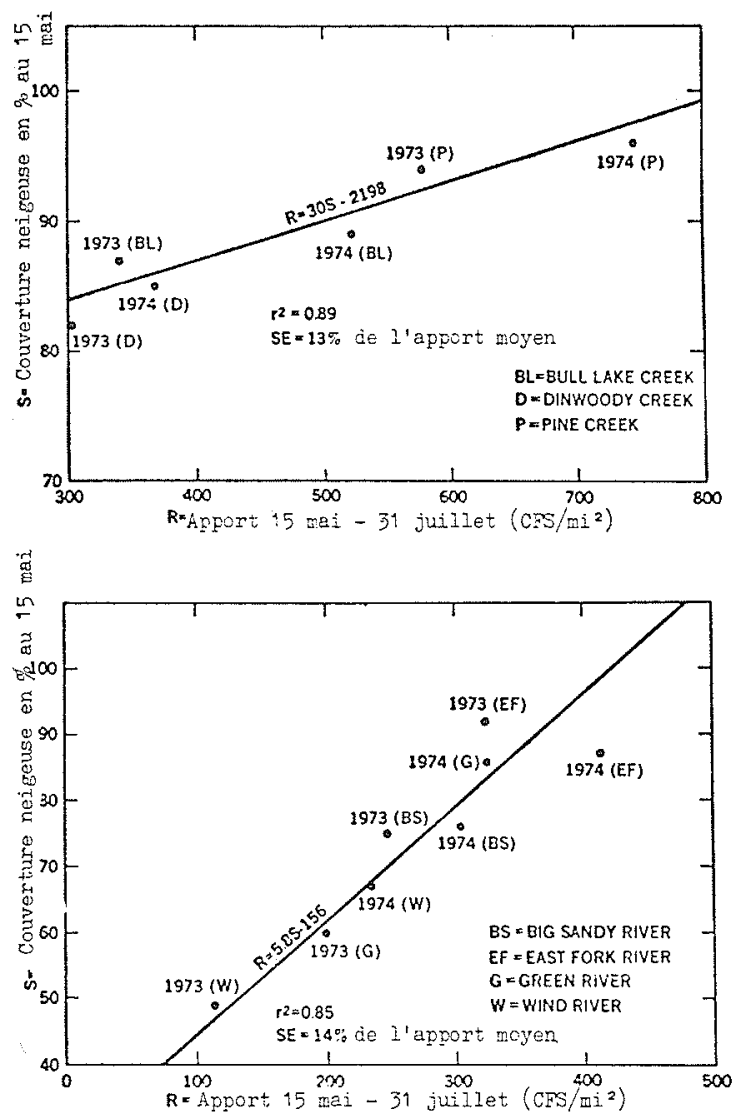

Figure 6

Mise à jour des prévisions pendant la fonte

L'idée est de suivre les variations de surface enneigée pour les relier aux volumes écoulés, en vue d'une prévision à beaucoup plus court terme (18 jours entre 2 passages de Landsat- 1 ou 9 jours entre un passage de Landsat-1 et un passage de Landsat-2).

Aul J.S. et Ffolliot P.F. (1975) traitent de la Black River, affluent de la Salt River dans le centre de l'Arizona (1450 $\mathrm{km}^{2}$ entre 1745 et $3533 \mathrm{~m}$ d'altitude). La variable à expliquer est le volume restant à écouler entre une photo et une date fixée considérée comme la fin de l'enneigement (exemple : 12 juin 1973).

Cette année-là, l'enneigement a commencé à décroître le 18 février et on a ajusté :

$Q_{j}=5,2 \times 10^{5}+2,7 \times 10^{5} \times X_{j}$

$X_{j}=$ superficie enneigée en $\mathrm{km}^{2}$ le jour $\mathrm{j}$

$Q_{j}=$ volume prévu entre le jour j et le 12 juin en $\mathrm{m}^{3}$

$r=.995$ avec $N=4$

La relation est encore vérifiée si l'on introduit 5 autres photos provenant de survols aériens.

La date de fin de la fonte ne peut être connue qu'à posteriori. La relation obtenue, outre son faible échantillonnage, correspond à une seule année : il est abusif de qualifier de corrélation une co-variation chronologique de 2 variables dont l'une est un cumul par définition monotone décroissant.
Thompson A.G. (1975) étudie le bassin de la Little Laramie River (404 km² entre 2300 et $3600 \mathrm{~m}$ d'altitude). Les photos utilisables (Landsat-1) sont au nombre de 15 réparties sur 1973,1974 et 1975 . La nébulosité plus forte dans le Wyoming que dans l'Arizona fait que nombre de photos furent inutilisables.

Une corrélation intéressante apparaît entre :

$Q_{j}=$ volume écoulé entre le $1^{\mathrm{er}}$ avril et le jour $\mathrm{j}$

$S_{j}=$ pourcentage d'enneigement le jour $\mathrm{j}$.

Toutefois, cette corrélation semble différer d'une année à l'autre, et pour la rendre homogène, l'auteur a pris :

$$
q_{j}=Q_{j} / V
$$

avec $V=$ volume total du $1^{\text {er }}$ avril au 31 juillet d'où la corrélation :

$$
\log _{10} q_{j}=2.04-1,565 S_{j} \quad r^{2}=.98 \quad N=11
$$

en ne considérant que les points pour lesquels $S_{j}$ est sensiblement inférieur à 100 . L'utilisation possible est : soit une estimation de l'apport saisonnier $V$, remise à jour à chaque photo, soit par différence avec $Q_{j}$, le volume restant à écouler.

A.G. Thompson constate que pour une évolution sensiblement voisine des \% d'enneigement en 1973 et 1974 , les 2 apports saisonniers sont dans le rapport de 7 à 9 ; c'est pourquoi il travaille en valeurs relatives.

Pour les courtes échéances, on est limité à la résolution des images ( 80 à $100 \mathrm{~m}$ pour Landsat) : il faut que l'enneigement ait suffisamment varié par rapport à la précision de la mesure. Une autre approche $\left({ }^{1}\right)$ consiste à

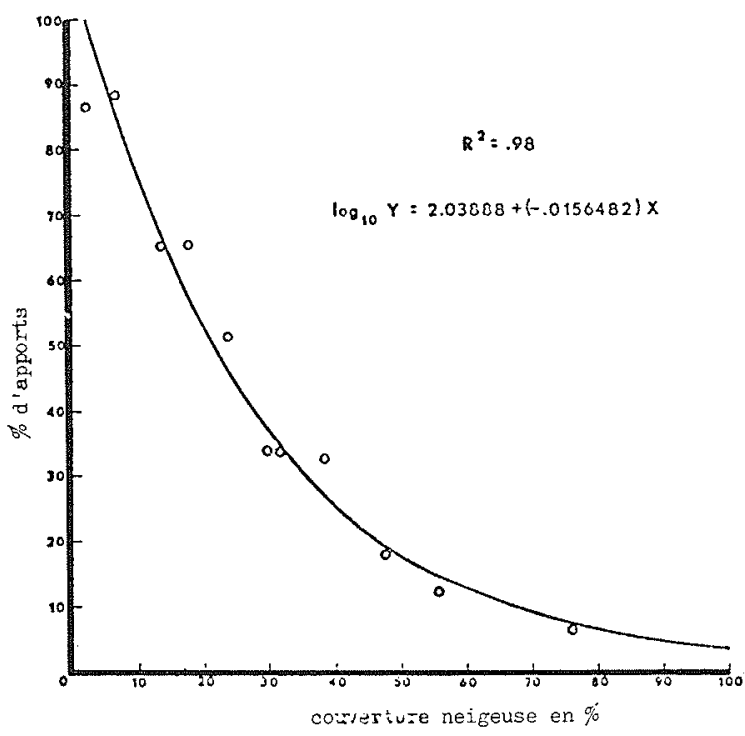

Figure 7 - D'après A.G. Thompson (1975).

(1) Walter Good - Institut Fédéral Suisse pour l'Etude de la Neige et des Avalanches (communication personnelle). 
analyser la forme du contour d'enneigement, qui serait un indice de l'équivalent en eau de la couche au voisinage du contour, de plus en plus aléatoire quand on s'en éloigne.

\section{Insuffisance de l'information surface enneigée}

Cette donnée ne prend pas en considération la variabilité de la répartition spatiale du dépôt qui résulte des facteurs synoptiques (type de temps lors des chutes de neige), mais aussi topographiques (orientation de versant et altitude).

Elle est sans doute plus représentative dans des climats continentaux ou semi-arides, où la fonte est commandée par un ensoleillement fort et régulier. Par contre, dans des climats tempérés, on peut très bien avoir, en fin de saison, chute de pluie et fonte à basse altitude donc réduction de la superficie enneigée, et chute de neige en altitude donc accroissement du stock.

Enfin, de toute façon, cette variable est inutilisable tant que les bassins considérés sont enneigés à $100 \%$, c'est-à-dire tant que la fonte n'a pas largement mordu sur le stock.

\section{Complémentarité avec les mesures au sol}

\section{Haute montagne}

Les mesures au sol, par sondage et pesée d'échantillon, du manteau neigeux en région montagneuse sont pratiquées depuis plus de 30 ans en Europe et aux EtatsUnis pour la prévision des apports saisonniers de fonte. La possibilité d'adapter chaque année, quelques mois à l'avance, l'utilisation des réservoirs aux apports attendus, présente un intérêt toujours actuel pour la production d'énergie et l'irrigation.

La représentativité de ces mesures ponctuelles, sur laquelle on peut à première vue s'interroger quand on voit la variabilité du dépôt de neige sous l'effet du vent et du relief, est attestée par les corrélations de .85 à .95 entre le volume d'apport avril-août et la valeur en eau de la couche en fin d'hiver en un nombre souvent très réduit d'emplacements sélectionnés et balisés : ces corrélations sont utilisées à partir de février dans les Alpes du Nord et les Pyrénées, dès janvier dans les Alpes du Sud (qui reçoivent leurs plus grosses chutes dans la première moitié de l'hiver), pour prévoir les apports de fonte de 50 bassins versants qui alimentent les usines hydroélectriques.

C'est en effet le plus tôt possible avant la fonte, quand la demande d'énergie est encore forte, qu'il faut profiter des accroissements connus des stocks de neige pour augmenter la production d'énergie des lacs et, c'est dans ce but qu'E.D.F. équipe les meilleures emplacements témoins d'enneigement avec le nivomètre à faisceau horizontal mobile qui transmet une fois par jour le profil complet de densité par décimètre, d'où la valeur en eau de la couche de neige. Ce dispositif est le seul qui donne une information complète (Fig. 8) sur la structure de la couche, structure qui intéresse tous les usagers de la neige (Vuillot et Guillot, 1968).

En fait, si ces mesures-témoins ponctuelles, entre 1500 et 2500 mètres d'altitude, sont bien représentatives du stock neigeux, ou du moins de sa valeur relative inter-annuelle, c'est surtout dans la période d'accumulation continue qui va de novembre à avril : elles constituent alors une information directe qui concurrence, et dans bien des cas surclasse, en tout cas complète utilement, l'évaluation indirecte calculée par le cumul, à partir de novembre, de la pluviométrie mesurée dans les vallées habitées voisines. Par contre, de mai à juillet, la représentativité de ces données ponctuelles se dégrade sans doute progressivement avec la fonte ; l'ablation est très influencée par les particularités locales d'exposition, tandis que le stock neigeux résiduel est de plus en plus concentré dans les congères et culots d'avalanches amassés dans les concavités du relief. Aussi c'est sans trop de regret que nous constatons l'altération de la couche par réverbération autour des pieds de nivomètre dans les dernières semaines de fonte.

Pour prévoir à court terme les variations du débit de fonte, connaissant la température et les précipitations, on a recours soit à une estimation physique de la fonte avec ses difficultés d'extrapolation selon l'exposition et l'altitude, soit plus modestement aux corrélations entre débits successifs et à l'explication statistique des écarts à la forme standard de la crue de fonte (analyse en composantes principales des apports hebdomadaires successifs). A la synthèse des deux approches, le suivi par satellite de la surface enneigée pourra peut-être utilement contribuer, à condition que la neige fraîche ne vienne pas trop souvent recouvrir les contours du vieux manteau. C'est dire que les chances de la télédétection sont meilleures en climat méditerranéen ou californien que dans le Nord des Alpes ou les Rocheuses canadiennes.

\section{Moyenne montagne}

En moyenne montagne, sur les grandes surfaces entre 800 et 1200 mètres du Jura et du Massif Central, plusieurs épisodes de quelques jours de dépôt et de fonte partielle ou totale peuvent se succéder chaque hiver. Dans ces régions, pour prévoir les variations du débit de crue pluvio-nivale, il faut suivre plusieurs fois par jour la valeur en eau du manteau de neige mouillée, dont la contribution à l'écoulement est tantôt positive (fonte), tantôt négative (précipitation en neige ou pluie retenue par la neige).

Dans cette tranche d'altitude nous utilisons le nivomètre à faisceau vertical fixe qui est bien adapté pour suivre les fluctuations de valeur en eau d'une couche de quelques décimètres car il ne risque pas d'altérer la couche de neige en cours de fonte (Fig. 9).

Dans ces régions à faible relief, les limites fluctuantes du manteau sont souvent mal connues même quand on a quelques informations au sol. C'est sans doute dans ces régions que les images fréquentes de satellite pourraient le plus aider à apprécier l'extension du manteau, d'où la transposition d'un versant à l'autre de la relation enneigement-altitude. La technique d'analyse des images 


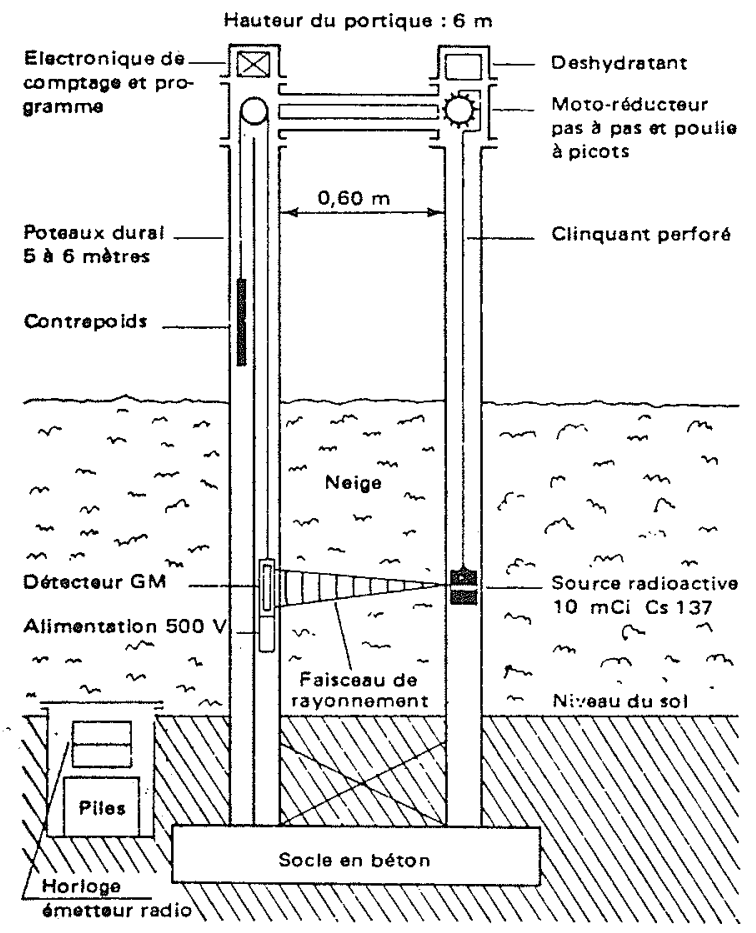

- Schéma du télénivomètre horizontał mobile

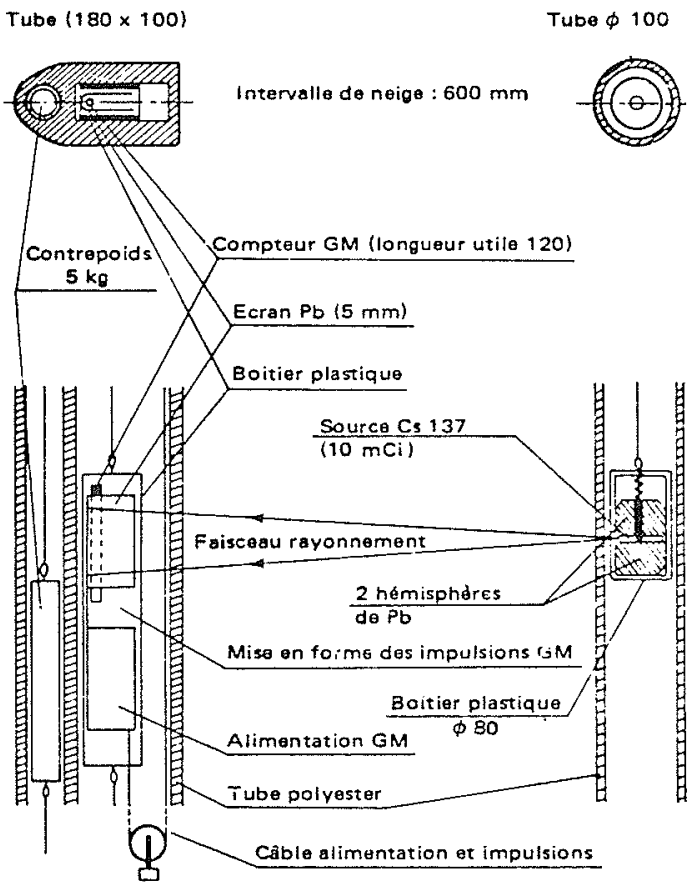

- Teleśnivomètre horizontal, coupe horizontale et verticale de l'ensemble de mesure

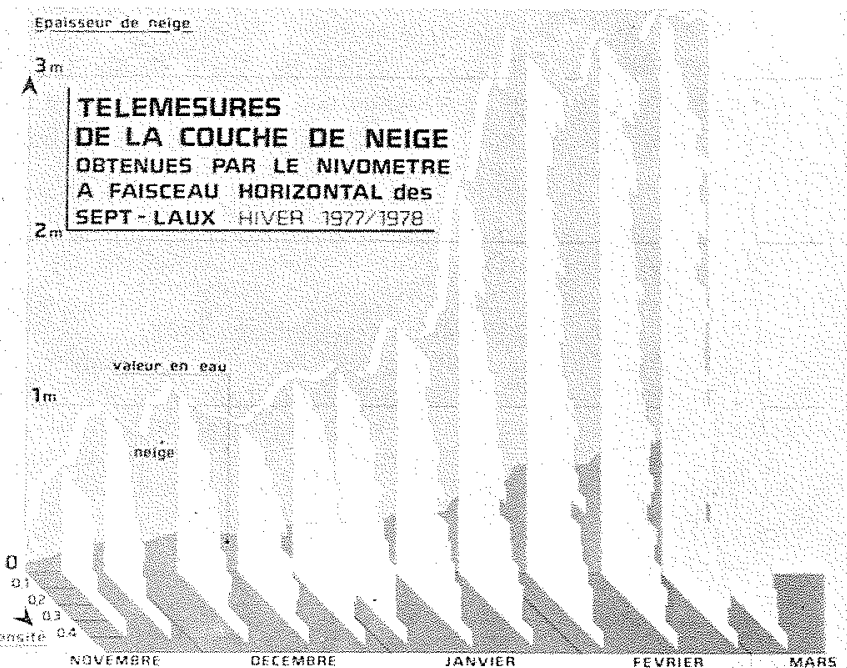

Profil de l'enneigement des Sept-Laux durant l'hiver 1977-1978.

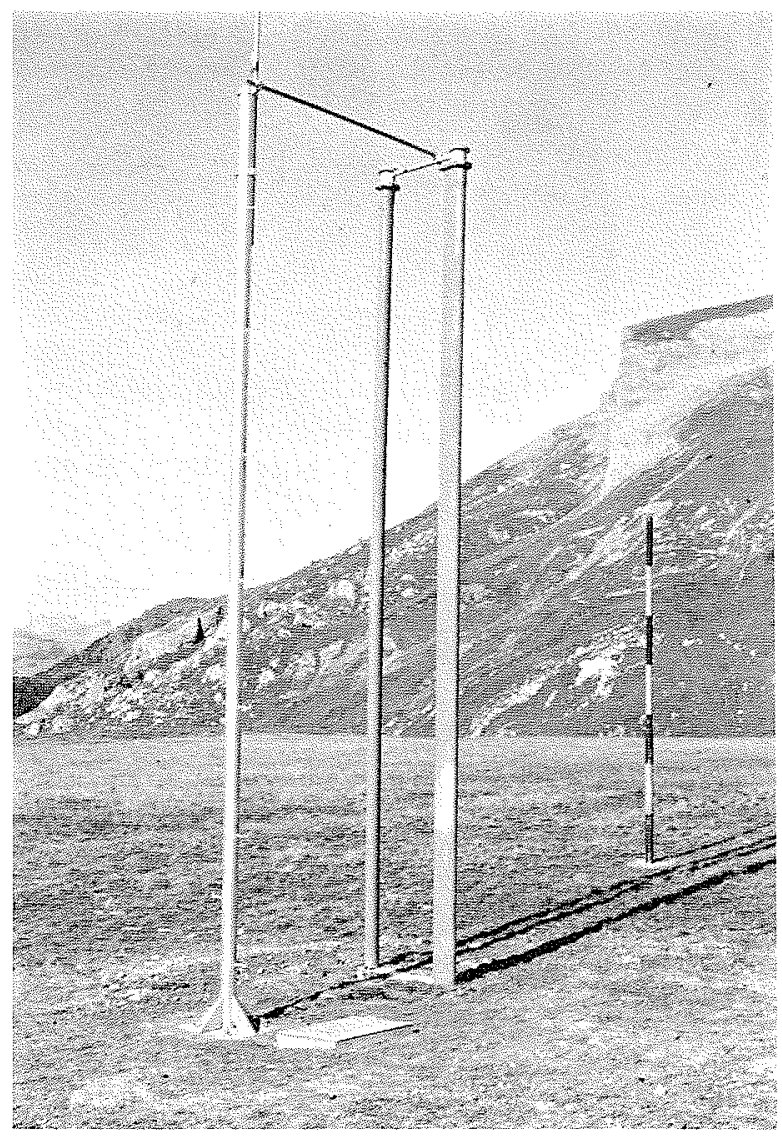

Le télénivomètre à faisceau horizontal mobile 


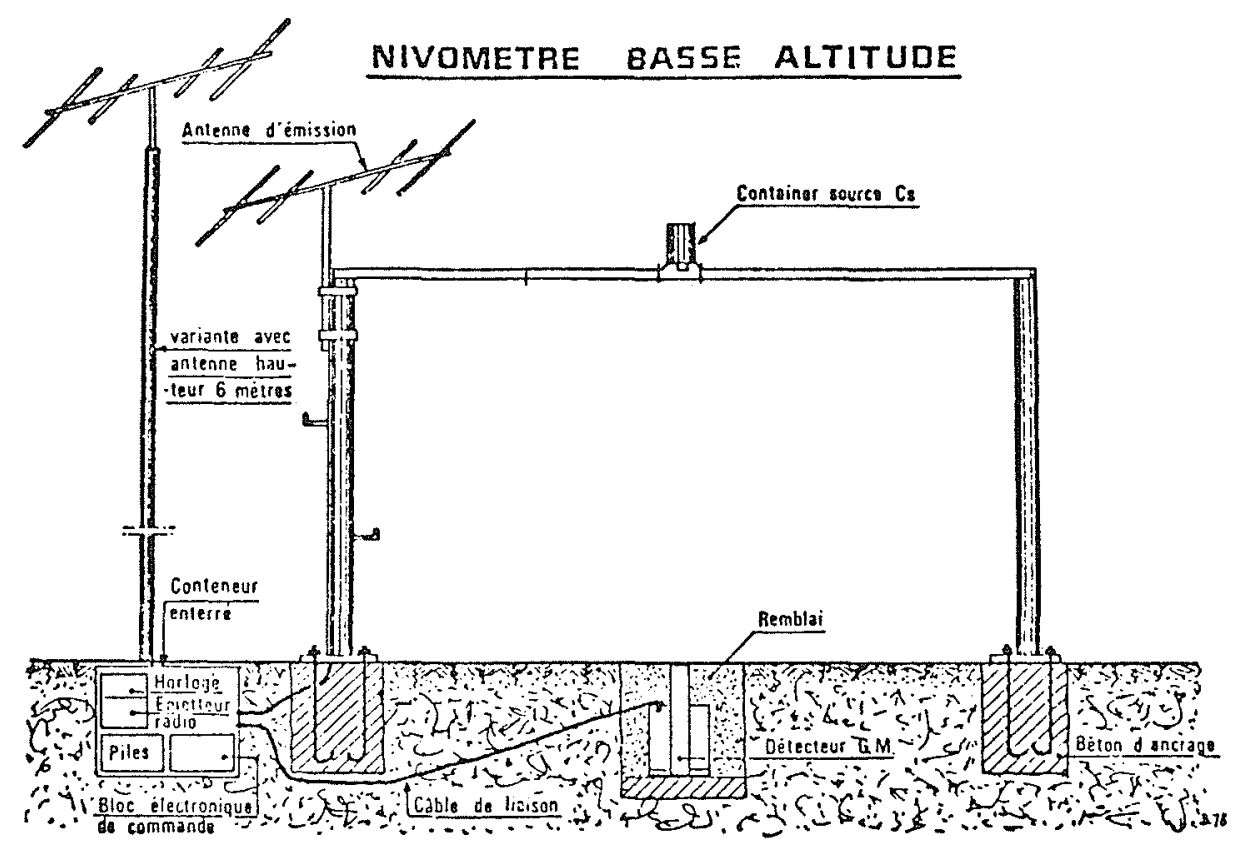

Figure 9 - Schéma du nivomètre à faisceau vertical fixe.

de NOAA-5 (histogramme de brillance) décrite par Langham et Power (1977) pour calculer les surfaces enneigées des bassins boisés à faible relief du bouclier canadien est peut-être transposable au Massif Central et au Jura.

\section{Plaines}

Dans les grandes plaines continentales, le total des précipitations d'hiver ne dépasse pas quelques décimètres de poudreuse redistribués par le vent : la représentativité des mesures ponctuelles est un problème qui tracasse depuis longtemps les hydrologues des pays de l'Est, si on en juge par le nombre de leurs publications sur le sujet. Une solution élégante appliquée dans ces pays depuis au moins 20 ans est la mesure de l'atténuation par la neige du rayonnement gamma émis par le terrain : dans les débuts, on notait la moyenne mesurée sur un long parcours à ski (6 compteurs Geiger-Müller portés à la ceinture), on procède maintenant en avion volant à basse altitude, avec un détecteur à scintillation de grande sensibilité. Cette méthode de télédétection n'est pas applicable en terrain accidenté, ni quand la valeur en eau dépasse 200 millimètres.

\section{Conclusion}

La télédétection à partir de satellites ne parâ̂t pas susceptible de remplacer les mesures au sol pour fournir la valeur en eau des stocks neigeux alpins ou pyrénéens en cours d'accumulation en hiver, c'est-à-dire pour calculer en temps utile les prévisions d'apports saisonniers de fonte. Dans l'avenir, et sous réserve d'amélioration substantielle de la fréquence et de la précision des vues, la télédétection pourra servir à améliorer la prévision à court terme par l'analyse des franges du manteau saisonnier en cours de fonte: formes de contour, albedo, température de surface.

Un certain espoir peut aussi être fondé sur la télédétection par les hyperfréquences (longueur d'onde entre 1 et $300 \mathrm{~mm}$ ) qui traversent les nuages, et peuvent donner l'épaisseur de neige sèche mais sont réfléchies par la neige mouillée.

Dans l'état actuel des moyens, la seule application de la télédétection qui peut nous apporter en France un complément utile d'information est l'image quotidienne des surfaces enneigées du Massif Central et du Jura, donnée par les satellites NOAA à orbite polaire, éventuellement complété par Meteosat.

\section{Bibliographie}

AUL J.S. and FFOLLIOTT P.F., (August 1975). - Use of Areal Snow Cover Measurements from ERTS-1 Imagery in Snowmelt-Runoff Relationships in Arizona, Rapport NASA SP-391 : Proceedings of the Workshop on Operational applications of Satellite Snowcover Observations South Lake Tahoe Calif., pp. 103-112.

GULATI T.D. (sept. 1972). - Role of snow and ice hydrology in India, Actes du Colloque A.I.S.H. de Banff, pp. 610-623, publication $n^{\circ} 107$ de l'A.I.S.H.

HAEFNER J. and ITTEN K.I., (1976), WMO. - Snow studies by satellites in Switzerland, Dep. of Geography, University of Zurich.

LANGHAM E.J. and POWER J.M., (oct. 25-27, 1977). - Remote sensing of snow by satellite, 2nd Conference on hydrometeorology, Toronto, édité par l'A.M.S., pp. 268-271.

MAC CLAIN E.P. and BAKER D.R., (1969). - Experimental Large scale snow and ice Mapping with composite minimum brightness charts, ESSA Tech. Memo, NESCTM-12.

MAC GINNIS D.F. (Aug. 1975). - A Progress Report on Estimating Snow Depth Using VHRR Data from NOAA Environmental Satellites, Rapport NASA SP-391: Proceedings of the Workshop on Operational applications of Satellite Snowcover Observations South Lake Tahoe Calif., pp. 313-324. 
MAC MILLAN M.C. and SMITH J.L. (Aug. 1975). - Remote Sensing of Snowpack Density Using Shortwave Radiation, Rapport NASA SP-391: Proceedings of the Workshop on Operational applications of Satellite Snowcover Observations South Lake Tahoe Calif., pp. 361-373.

O'BRIEN H.W. and MUNIS R.H., (1975). - Red and nearinfrared spectral reflectance of Snow CRREL Research, report 332.

O'BRIEN H.W. and MUNIS R.H., (1977). - Observations of the ultravioleted reflectance of Snow, CRREL, report 77-27.

PERRIN de BRICHAMBAUT $\mathrm{Ch}$. et LAMBOLEY G., (janv. 1969). - Le rayonnement solaire au sol et ses mesures, Cahiers AFEDES, $\mathrm{n}^{\circ} 1$.

RANGO A. and SALOMONSON V.V. (Aug. 1975). - Employment of Satellite Snowcover Observations for Improving Seasonal Runoff Estimates, Rapport NASA SP-391: Proceedings of the Workshop on Operational applications of Satellite Snowcover Observations, South Lake Tahoe Calif., pp. 157-174.

RANGO A., (1976). - Results of NASA Snow studies, Summary Paper - International Working Seminar on snow studies by satellites, WMO Genève.
RANGO A. and SALOMONSON V.V., (feb. 1977). - Seasonal Stream flow Estimation in the Himalayan Region Employing Meteorological Satellite Snowcover Observations, Water Ressources Research, Vol. 13, $\mathrm{n}^{\circ}$ 1, pp. 109-112.

SEIFERT R.D. et al., (Aug. 1975). - Operational Applications of NOAA-VHRR Imagery in Alaska, Rapport NASA SP-391: Proceedings of the workshop on Operational applications of Satellite Snowcover Observations South Lake Tahoe Calif., pp. 143-155.

THOMPSON A.G., (Aug. 1975). - Utilization of Landsat Monitoring Capabilities fo Snowcover Depletion Analysis, Report NASA SP-391: Proceedings of the Workshop on Operational Applications of Satellite Snowcover Observations South Lake Tahoe, Calif., pp. 113-127.

VUILLOT M. et GUILLOT P., (déc. 1968). - Le télénivomètre à faisceau horizontal mobile, Bulletin de l'A.I.S.H., $\mathrm{n}^{\circ}$ XIII 4 , pp. 47-70.

WMO (oct. 1976). - Final Report of the International Working Seminar on Snow Studies by Satellites - Geneva 19/22 october 1976.

\section{Discussion}

Le Président remercie les auteurs et demande s'il ne serait pas souhaitable de pouvoir appliquer la télédétection à la mesure de la température de la neige. On sait, précise-t-il, qu'elle varie dans l'espace et dans le temps. La connaissance de cette variation, naturellement en dessous du zéro ! et celle de la température de l'air seraient précieuses pour la prévision de la fonte, done pour la gestion des centrales hydroélectriques, surtout en prévision à court terme lorsqu'il s'agit d'exploiter des bassins de compensation et des stations de pompage.

M. OBLED. - Dans l'état actuel, la télédétection pourrait fournir des photos "instantanées" de la température de surface du manteau de neige, par thermométrie infrarouge (domaine où la neige est un corps noir presque parfait). Malheureusement, cette valeur est plafonnée à $0^{\circ}$ par le changement d'état, ce qui ne permet pas de déterminer le taux de fonte en cas de bilan positif. De plus la neige étant un corps assez isolant, sa température de surface présente de grandes fluctuations et un faible temps de réponse (Cf. le graphique d'après Isyunov) ce pour des variations de flux assez petites.

La surface qui est à $0^{\circ}$ peut être un index hydrologique intéressant. Toutefois, cela suppose que le manteau sous jacent ne contienne pas un stock de frigories important, ce qui peut arriver. D'autre part, la liaison avec les volumes journaliers écoulés sera liée aux types de temps, car les phénomènes dominants dans le bilan ne sont pas les mêmes par temps clair ou par temps couvert et perturbé. Dans ce dernier cas, fort intéressant hydrologiquement, la télédétection par satellite est malheureusement impossible à cause des nuages.

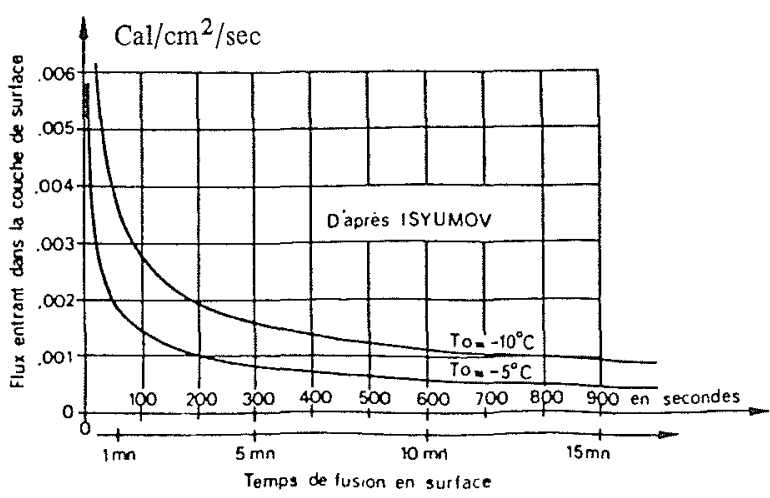

Temps nécessaire à l'apparition d'une température nulle à la surface d'une couche, initialement à $T_{0}$, et recevant un flux positif $q_{0}$ 


\section{Abstract \\ Satellite pictures and snow measurements}

This partly bibliographical study starts with a review of satellites transmitting useful data for snow surveys and draws attention to difficulties experienced in converting raw data into usable information (e.g. extent of snow-covered areas). Examples are then described of reports published by mostly North American authors, in which satellite data are related to hydrological variables. Most of the information originates from a NASA seminar at South Lake Tahoe in August 1975 and a WMO seminar in Geneva in October 1976, on the subject of "Satellite surveys of snow cover for hydrological forecasting". This study ends with a discussion of present use of groundbased measurements by "Electricité de France" for forecasting seasonal precipitation supplies and the types of additional service teledetection could provide.

It seems unlikely that teledetection from satellites will ever replace ground-based measurements as a means of evaluating the water equivalent of the snow burden in the Alps or Pyrenees while it is accumulating in witner, i.e. calculating probable snow melt inflows sufficiently far ahead. Satellite pictures of snow-covered areas do not become representative of the actual storage volume until several weeks after the snow starts to melt, i.e. not before the 15th May in the Alps, whereas Electricite de France forescasts from ground-based measurements become effective as from the lst February. The economic importance of such advance notice for water power storage management is the reason why such considerable efforts are being made to automate daily snow depth telemeasurements (the remote-indicating snow gauge with a moving horizontal beam provides a complete decimetre-by-decimetre density profile).

In future, and on the assumption that the frequency and accuracy of satellite pictures will substantially improve, teledetection should provide an effective means of improving short-term forecasts by analysis in the visible and infra-red ranges of the fringe areas of the seasonal snow cover while it is melting: contours, albedo, surface temperature.

Hyperfrequency teledetection at wavelengths from $1 \mathrm{~mm}$ to $300 \mathrm{~mm}$ also seems to offer reasonably hopeful prospects; such frequencies pass through clouds and can provide information on the depth of dry snow, but are reflected by wet snow.

With existing satellites the only application of teledetection capable of providing useful information, for observation of the short-term behaviour of floods originating from snow and/or rain, is the daily picture of snow-covered areas without pronounced relief features between altitudes of $800 \mathrm{~m}$ and $1200 \mathrm{~m}$ in the Massif Central and Jura mountains from NOAA satellites on a polar orbit, and perhaps also METEOSAT. 